\title{
The In Vitro and In Vivo Activity of Ciprofloxacin
}

Yasmin J Drabu, M.B. Ch.B., D.C.H., M.R.C.Path, Shaheen Mehtar, M.B., B.S., M.R.C.Path, Patricis H Blakemore, F.I.M.L.S., London, United Kingdom

http://dx.doi.org/10.5915/21-1-5517

\begin{abstract}
The third generation quinolones represent a new development and a possible alternative to the more convenrional B-lactam and aminoglycosides for the management of difficult nosocomial infections. This study compared the in vitro and in vivo antibacterial activity of ciprofloxacin, a new fluroquinolone. The in vitro anlibacterial activity of ciprofloxacin was compared with three quinolones, three aminoglycosides and three cephalosporins against 500 clinical isolates, including 100 gentamicin and 30 nalidixic acid resistant gram negative organisms. The latter were also tested against amifloxacin, ofloxacin and enoxacin. Minimum inhibitory concentration (MICs) were performed by agar dilution method using a final inoculum of $10^{4}$ colony forming units (CFU) $/ \mathrm{ml}$. The results showed that ciprofloxacin was the most active drug against aerobic gram negative bacteria. Aminoglycoside and cephalosporin resistance did not affect the MIC 90 , however resistance to nulidixic acid showed a four fold increase in MIC 90 of all the quinolones. The efficiency and safety of ciprofloxacin was determined in an open prospective study in 57 hospitalized patients with infections caused by multiresistant gram-negative bacteria. A variable dosage was used, dependent on the site of infection. Clinical and bacteriological success occurred in 49 patients which included 12 bacteremias. Eight patients were classed as failures; although clinical improvement occurred, the organisms persisted to the end of therapy. Persistence was associated with presence of prostheses. Two patients had minor side effects. We conclude that ciprofloxacin has good in vitro and in vivo activity against a wide range of aerobes including multi-resistant gram negative bacteria.
\end{abstract}

Key words: In vivo and in vitro activity, ciprofloxacin, quinolones, multi-resistant gram negatives.

The quinolone carboxylic acid derivatives of nalidixic acid represent a new development in synthetic antimicrobial therapy and are a possible alterative to the more conventional B-lactam and aminoglycosides for the management of difficult nosocomial infections. This study compared the in vitro antibacterial activity of ciprofloxacin, a new fluroquinolone with twelve antibiotics; six quinolones, three aminoglycosides and three cephalosporins. The in vivo safety and efficacy of ciprofloxacin was determined in the treatment of patients with infections caused by multi-resistant gramnegative bacteria.

From the Department of Microbiology

North Middlesex Hospital

Reprint requests: Dr. Y J Drabu

Deparliment of Microbiology

Norlh Middlesex Hospital

London NI8 IQX, United Kingdom
I. In vitro studies:

Materials and methods

The antibacterial activity of ciprofloxacin was compared with three quinolones (pefloxacin, norfloxacin and nalidixic acid), three aminoglycosides (gentamicin, netilmicin and amíkacin) and three cephalosporins (cefuroxime, cefoxitin and ceftazidime) against 500 clinical isolates. Thirty nalidixic acid resistant (Minimum Inhibitory Concentration (MIC) $>16 \mathrm{mg} / \mathrm{L}$ enterobacteriacease were also tested against three other quinolones (enoxacin, amifloxacin and ofloxacin).

The clinical isolates were collected over a period of four years and included 400 aerobic gram negative and 100 aerobic gram positive organisms, identified by standard laboratory methods including API $20 \mathrm{E}$ for enterobacteriacease. These included 121/275 gentamicin resistant (MIC $>16 \mathrm{mg} / \mathrm{L}$ ) and $30 / 275$ nalidixic acid resistant (MIC $>16 \mathrm{mg} / \mathrm{L}$ ) enterobacteriacease, 5/24 gentamicin resistant Ps. aeruginosa, $21 / 30$ B-lactamase producing Hemophilus influenzae, $6 / 15 \mathrm{~B}$-lactamase producing 
Table 1. Minirsurn inhibitory concentrations (MIC) of 10 antibiotics versus 500 clinical isolates

\begin{tabular}{|c|c|c|c|c|c|c|}
\hline $\begin{array}{l}\text { Antiblotic } \\
\text { (MIC 90) }\end{array}$ & $\mathrm{S}$ & $\mathbf{R}$ & $\begin{array}{c}\text { Entero- } \\
\text { bacter }(\mathbf{1}) \\
\mathbf{n}=\mathbf{2 7 5}\end{array}$ & $\begin{array}{c}\text { Ps. spp (2) } \\
n=50\end{array}$ & $\begin{array}{l}\mathrm{FgN}(3) \\
\mathrm{n}=75\end{array}$ & $\begin{array}{c}\text { Gram } \\
\text { pos. (4) } \\
n=100\end{array}$ \\
\hline $\mathrm{Cip}$ & 2 & 4 & 0.5 & 8 & 0,03 & 4 \\
\hline Pef & 4 & 8 & 1 & 8 & 0.25 & 16 \\
\hline Nor & 8 & 16 & 2 & 16 & 0.25 & 16 \\
\hline $\mathrm{Nal}$ & 8 & 32 & 128 & 128 & 4 & 128 \\
\hline Gent & 4 & 8 & 128 & 128 & 8 & 64 \\
\hline Net & 4 & 32 & 64 & 128 & 4 & 64 \\
\hline Amik & 16 & 32 & 32 & 128 & 32 & 128 \\
\hline Cxin & 8 & 32 & 128 & 128 & 8 & 128 \\
\hline Fox & 8 & 32 & 64 & 128 & 8 & 128 \\
\hline $\mathrm{Caz}$ & 8 & 32 & 2 & 4 & 0.125 & 128 \\
\hline
\end{tabular}

(1) Enterobacteriaceae

(2) Pseudomonas sp.

(3) Fastidious gram negatives (Hemophilus influenzae, Branhamella catarrhalis, Neisseria sp.)

(4) Gram-positive (Graph sp, Strep specis).

Cip $=$ ciprofloxacin

Pef $=$ pefloxacin

Nor $=$ norfloxacin

$$
\begin{array}{ll}
\text { Nal }=\text { nalidixic acid } & \text { Amik = amikacin } \\
\text { Gent = gentamicin } & \mathrm{Cxm}=\text { cefuroxime } \\
\text { Net = netilmicin } & \text { Fox }=\text { cefoxitin } \\
& \mathrm{Caz}=\text { ceftazidime }
\end{array}
$$

Neisseria gonorrhoeae, 18/44 methicillin resistant and 7/44 gentamicin resistant Staph. aureus.

The MIC of the antibiotics were determined by agar plate dilution method using Oxoid diagnostic sensitivity agar $\mathrm{pH} 7.3$ supplemented with $7 \%$ lysed horse blood. Ten percent horse serum was added to support the growth of Streptococcus pneumoniae. A final inoculum of $10^{4} \mathrm{cfu} / \mathrm{ml}$ was delivered via a multipont inoculator (Denley Tech Ltd.). The organisms were incubated for 24 hours aerobically at $37^{\circ} \mathrm{C}$ except for $H$. influenzae, Branhamella catarrhalis and campylobacter which were incubated in $10 \% \mathrm{Co}_{2}$. The Oxford strain of Staph. aureus and E. coli NCTC 10418 were used as controls. The MIC of the antibiotic against all organisms were defined as the lowest concentration of drug which inhibited visble growth.

\section{Results}

Table 1 is a summary of the MIC results obtained for the 500 clinical isolates. Table 2 shows the activity of the six new quinolones against 30 nalidixic acid resistant (MIC $>16 \mathrm{mg} / \mathrm{L}$ ) enterobacteriaceae.

Ciprofloxacin is the most active quinolone and has excellent activity against fastidious gram-negatives, enterobacteriaceae and Ps. aeruginosa, comparable to ceftazidime; aminoglycoside and cephalosporin resistance does not affect the MIC 90 , however resistance to nalidixic acid shows a four fold increase in MIC 90 of ciprofloxacin and cross resistance with other quinolones. It has moderate activity against gram positive organisms.
Table 2. Minimum inhibitory concentrations of six quinolones against nalidixic acid-resistant enterobacteriaceae, $\mathrm{n}=30$

\begin{tabular}{llrrrrl}
\hline Antibiotic & cip & per & nor & eno & ami & oflox \\
\hline MIC $50 \mathrm{mg} / \mathrm{L}$ & 0.125 & 2 & 2 & 2 & 2 & 0.5 \\
MIC $90 \mathrm{mg} / \mathrm{L}$ & 4 & 16 & 16 & 16 & 16 & 8 \\
\hline cip = ciprofloxacin & eno = enoxacin \\
pef = pefloxacin & ami = amifloxacin \\
nor = norfloxacin & oflox = ofloxacin
\end{tabular}

\section{In vivo studies:}

\section{Materials and methods}

An open prospective study was undertaken to assess the clinical and bacteriological efficacy of oral ciprofloxacin in the treatment of patients infected with multi-resistant gram negative bacteria. These included enterobacteriaceae resistant to ampicillin, sulphonamide and trimethoprim (on disc sensitivity testing by Stoke's method) and to gentamicin (MIC $>16 \mathrm{mg} / \mathrm{L}$ ). All the isolates treated were fully sensitive to ciprofloxacin (MIC $<2 \mathrm{mg} / \mathrm{L}$ ). Fifty-seven hospitalised patients ( 38 men and 19 women) were treated; the ages ranged from 18-93 years (mean age, 73).

The infections treated included bacteremia (12), urinary tract infection (37), bone (3), skin and soft tissue (5). Local ethical committee approval and informed consent from the patient or next of kin were obtained. Pregnant or lactating women, patients under 16 years of age and patients with hepatic or renal dysfunction were excluded from the study.

A variable dosage regime was used according to the site and severity of infection. Ten patients were treated with $100 \mathrm{mg}$ given 8 hourly, 13 with $250 \mathrm{mg}$ 12 hourly, 11 with $250 \mathrm{mg} 8$ hourly and 23 with 500 $\mathrm{mg} 12$ hourly. Clinically, radiological and bateriological parameters were used to evaluate response to therapy. Satisfactory clinical response was documented if improvement in signs and symptoms of infection occurred. Bacteriological cure was defined as elimination of the organism from culture sites on completion of therapy and also on follow up at seven days (and one month after therapy for urinary tract infections). Hematological and biochemical parameters were monitored throughout.

\section{Results}

Thirty seven patients, 15 of whom were catheterized were treated for urinary tract infection, a total of 42 strains were isolated, six patients and associated skin and soft tissue infection. The isolates included Klebsiella oxytoca (10), Klebsiella pneumoniae (5), E. coli (6), Prov. stuartii (7), Ent. cloacae (5), Citrobacter freundii (5), Prot. mirabilis (2), 
Morganella morganii (I), and Ps. aeruginosa (I). Thirty-three patients were treated successfully and 38 of the $\mathbf{4 2}$ isolates were eradicated.

Associated soft tissue infections in six patients were treated successfully. Three patients were treated for bone infection, which included a Brodie's abscess infected with Salmonella munchen, and two total hip replacement infected with $E$. coli. The latter two persisted after 60 and 120 days therapy with ciprofloxacin. Five patients including a petient with salpingitis caused by B-lactamase producing $N$. gonorrhoeae and two patients with arterial infection caused by $P S$. aeruginosa were treated (the latter again showed persistence after 60 days of therapy, although clinical improvement had occurred).

Twelve cases of basteremia were treated successfully and included E. coli (4) Ps. aeruginosa (2). A patient with Salmonella typhimurium (resistant to ampicillin, contrimoxazone and chloramphenicol) septicemia was treated with 4 days of intravenous ciprofloxacin and then changed to oral ciprofloxacin, recovered completely after 14 days therapy.

Sixty-eight bacterial strains were isolated from 57 patients; all but eight were eliminated with ciprofloxacin therapy. Bacterial persistence in three patients was associated with urinary tract infection, in catheterised patients. They were caused by Ps. aeruginosa and two by $E$. coli, in patients with prostheses. Four organisms; Klebsiella (2) and Pseudomonas (2) demonstrated an increase in ciprofloxacin MIC during therapy. The former was resistant to ampicillin, sulphonamide, trimethoprim and nalidixic acid.

Two patients suffered nausea and vomiting as side effects, and another had transient rise in liver function tests during therapy, which returned to normal at the end of therapy. The overall results showed $93 \%$ clinical success rate and $86 \%$ bacterial eradication rate.

\section{Discussion}

This study showed that in vitro ciprofloxacin was the most active quinolone against aerobic gram negative coliforms. It has moderate activity against Pseudomonas species and the gram positive organisms and is inactive against the anaerobic organisms.' There is no cross resistance with the B-lactam antibiotics or with the aminoglycosides. Nalidixic acid resistance raises the MIC by 4 -fold for all the quinolones. ${ }^{2}$

In vivo it is well tolerated and effective in treating multi-resistant gram negative infections including bacteraemias. ${ }^{3,4}$ Persistence of the organism after therapy was noted when the infection was associated with a foreign body e.g. catheter or prosthesis, or initial nalidixic acid resistance. Pseudomonas aeruginosa, a difficult organism to treat, requiring a combination of B-lactam and aminoglycoside therapy can be treated effectively with high dose oral ciprofloxacin.

Ciprofloxacin as a representative of the quinlone group, heralds a new era in antibiotic therapy for multi-resistant gram negative coliforms.

\section{References}

1. Chin, Nai-Xun, Neu, H C: Ciprofloxacin, a quinolone carboxylic acid compound active against aerobic and anaerobic bacteria. Antimicrobial agents. Chemotherapy 1984; 25:319-326.

2. Smith, J T: Mutational resistance to 4-quinolones antibacterial agents. Europ J Clin Microbiol 1984; 3:347-350.

3. Arcieri, G, Griffith, E, Gruenwaldt, $G$ et al: Ciprofloxacin: an update on clinical experience. Med 1987; 82 (Suppl. 4A): 381-386.

4. Gasser, T C, Graversen, P D, Madsen, P O: Treatment of Complicated Urinary tract Infection with ciprofloxacin. Am J Med 1987; 82 (Suppl. 4A): $278-280$.

\section{Erratum:}

In the article by Malik-Afzali and Chamsa, entitled "Neonatal Motality Due to Tetanus in Iran" (JIMA 1988;20:104-110), the following corrections should be noted:

Page 104, Abstract, line 6: over 1,000 children should read: over 10,000 children.

Page 105, Table 1, column "Live Birth":total 44,870-should read : 144,870 .

Page 105, text below map, line 1, read: group with should read: group 1 with . . .

Page 105, text below map, line 3: . . 75 per $1,000-$ should read 75 per $100^{2}$.

Page 107, Table 5, title row: "1987' - should read "total". 